\title{
Model Elastomeric Networks Prepared from Poly(caprolactone)
}

\author{
Po-Hou SUNG and James E. MARK \\ Department of Chemistry and the Polymer Research Center, \\ University of Cincinnati, Cincinnati, Ohio 45221, U.S.A.
}

(Received May 19, 1980)

\begin{abstract}
Model networks of the polyurethane-type were prepared by end-linking hydroxyl-terminated poly( $\varepsilon$-caprolactone) chains with an aromatic triisocyanate. The chains had number-average molecular weights $M_{n}$ in the range $570-1950 \mathrm{~g} \mathrm{~mol}^{-1}$, and the reactions were carried out in the undiluted state. The trifunctional networks thus obtained were studied with regard to their stress-strain isotherms in both the unswollen and swollen states, in elongation at $25^{\circ} \mathrm{C}$. Values of the high deformation modulus fell between the limits predicted for the non-affine deformation of a "phantom" network and the affine deformation of a network in which the crosslinks are firmly embedded in their surroundings. As expected, the values of the low deformation modulus were much closer to the affine limit.

KEY WORDS Model Networks / Poly(caprolactone) / Elastomeric Polyurethanes / Stress-Strain Isotherms / Mooney-Rivlin Constants / Phantom Networks / Affine Deformations /
\end{abstract}

"Model" polymer networks are those which have been prepared with careful control over the crosslink functionality $\phi$ and the average molecular weight $M_{\mathrm{c}}$ between cross-links. ${ }^{1}$ This is usually achieved by synthesizing bifunctionally-terminated chains, measuring their number-average molecular weights $M_{n}$, and then end-linking them essentially quantitatively with a cross-linking reactant of functionality three or greater. The resulting networks thus have cross-links of functionality equal to that of the cross-linking agent, and values of $M_{\mathrm{c}}$ corresponding to the values of $M_{n}$ of the chains prior to their end-linking into the network structure.

Elastomeric networks of this type may be used to test the molecular theories of rubber-like elasticity, ${ }^{2-6}$ particularly with regard to the very important modulus in elongation. The values predicted for this modulus depend on how closely the elongation deformation approaches the idealization of an affine deformation, in which the displacements of the cross-links are assumed to be a simple linear function of the macroscopic strain. Comparisons of experimental and theoretical values of the modulus can thus be used to elucidate the nature of the elastic deformation in the regions of high and low elongations. ${ }^{1,7}$

Such comparisons between experiment and theory have been carried out in the present investigation, using model networks of the polyurethane type $\mathrm{e}^{7-11}$ prepared by end-linking poly $(\varepsilon-$

caprolactone) (PCL) chains ${ }^{12}\left[\left(\mathrm{CH}_{2}\right)_{5} \stackrel{\mathrm{O}}{\mathrm{CO}}\right]$ with a specially prepared triisocyanate. This polymer is crystallizable, with a (maximum) melting point of approximately $60^{\circ} \mathrm{C}^{13}$ Strain-induced crystallization $^{14}$ was suppressed, however, at least for small and moderate deformations, by incorporation of a non-volatile diluent in the networks during the stress-strain measurements. ${ }^{15}$

\section{EXPERIMENTAL DETAILS}

The PCL bifunctional polymers employed were four samples generously provided by Dr. J. V. Koleske of the Union Carbide Corporation, S. Charleston, WV. Their designations were NIAX Polyols PCP 200, 210, 230, and 240, with specified approximate values of $M_{n}$ of $530,830,1250$, and $2000 \mathrm{~g} \mathrm{~mol}^{-1}$, respectively. More accurate values 
Table I. Elastomeric properties and structural characteristics of the poly( $\varepsilon$-caprolactone) networks

\begin{tabular}{|c|c|c|c|c|c|c|c|}
\hline $10^{-3} M_{n}$ & & & Isotherm & $2 C_{1}$ & $2 C_{2}$ & & \\
\hline $\mathrm{g} \mathrm{mol}^{-1}$ & & & designation & $\mathrm{N} \mathrm{mm}^{-2}$ & $\mathrm{~N} \mathrm{~mm}^{-2}$ & & \\
\hline \multirow[t]{3}{*}{0.570} & 0.999 & 1.000 & 1 & 2.47 & 1.23 & 0.530 & 0.794 \\
\hline & & 0.775 & 2 & 2.87 & 0.610 & 0.615 & \\
\hline & & 0.558 & 3 & 2.98 & 0.491 & 0.639 & \\
\hline \multirow[t]{3}{*}{0.910} & 0.992 & 1.000 & 4 & 2.15 & 0.375 & 0.740 & 0.871 \\
\hline & & 0.853 & 5 & 2.06 & 0.424 & 0.709 & \\
\hline & & 0.459 & 6 & 2.19 & 0.152 & 0.754 & \\
\hline \multirow[t]{3}{*}{1.31} & 0.984 & 1.000 & 7 & 1.40 & 0.779 & 0.698 & 1.09 \\
\hline & & 0.766 & 8 & 1.39 & 0.802 & 0.693 & \\
\hline & & 0.394 & 9 & 1.33 & 0.664 & 0.663 & \\
\hline \multirow[t]{4}{*}{1.95} & 0.972 & 1.000 & 10 & 0.957 & 0.850 & 0.718 & 1.36 \\
\hline & & 0.623 & 11 & 0.861 & 1.01 & 0.646 & \\
\hline & & 0.450 & 12 & 0.788 & 1.05 & 0.591 & \\
\hline & & 0.402 & 13 & 0.800 & 0.992 & 0.600 & \\
\hline
\end{tabular}

a Volume fraction of polymer incorporated in the network structure by the end-linking reaction.

b Volume fraction of polymer in the network (swollen with phenyl acetate) during the stress-strain measurements at $25^{\circ} \mathrm{C}$.

were obtained using a Knauer 1001-A vapor pressure osmometer which had been calibrated using solutions of highly purified benzil. All measurements were carried out at $36^{\circ} \mathrm{C}$, with chloroform as solvent. The concentrations of the PCL solutions ranged from approximately 0.3 to $5.0 \mathrm{~g} \mathrm{dl}^{-1}$, and the resulting values of $10^{-3} M_{n}$ are given in the first column of Table I.

These hydroxyl-terminated chains were endlinked using the aromatic triisocyanate

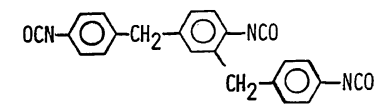

which had been specially prepared and generously provided by Dr. D. H. Chadwick of the Mobay Chemical Company, New Martinsville, WV. The urethane end-linking is an addition reaction, which may be represented schematically as

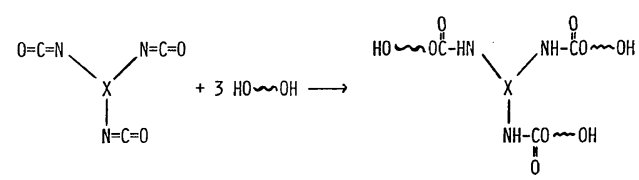

Both the polymer and the triisocyanate were exhaustively dried by heating under vacuum. Amounts of the two components corresponding to stoichiometric equivalence of $\mathrm{OH}$ and $\mathrm{NCO}$ groups were then mixed and placed into Teflon-lined aluminum molds. The end-linking reaction was then carried out under vacuum at $95^{\circ} \mathrm{C}$, for $50 \mathrm{~h}$, as described previously.

The resulting networks were extracted with gently stirred benzene for $6 \mathrm{~h}$ at $60^{\circ} \mathrm{C}$ and were then dried. The soluble portion amounted to approximately $1 \%$ of the original weight of a network; the precise values of $v_{2 \mathrm{C}}$, the volume fraction of polymer incorporated in the network structure, are given in the second column of the Table. The stress-strain isotherms were obtained on strips cut from the various network sheets, using standard techniques, ${ }^{16,17}$ at a temperature of $25^{\circ} \mathrm{C}$. For each polymer, one isotherm was determined in the unswollen state, but all of the others in the swollen state, in order to suppress strain-induced crystallization. The diluent used for this purpose was phenyl acetate; its relatively high boiling point $\left(196^{\circ} \mathrm{C}\right)$ assured only negligible losses of diluent during the stress-strain measurements at $25^{\circ} \mathrm{C}$. The extent of 
dilution was characterized by $v_{2}$, the volume fraction of polymer in the swollen network. The values employed are given in column three of the Table. The strips had unswollen cross-sectional areas $A^{*}$ of approximately $3 \mathrm{~mm}^{2}$, and the lengths of the central test portions of the strips were approximately $20 \mathrm{~mm}$. Stress-strain measurements were made using a sequence of increasing values of the elongation or relative length of the sample $\alpha=L / L_{\mathrm{i}}$, with some inclusions of values out of sequence to test for reversibility. Values of the elastic force $f$ were recorded only after they had become sensibly constant, and the elongation was generally increased to the rupture point of the sample.

\section{RESULTS AND DISCUSSION}

The stress-strain data were treated in the usual manner. ${ }^{16-18}$ Specifically, the values of the elastic modulus or reduced stress,

$$
\left[f^{*}\right] \equiv f v_{2}^{1 / 3} /\left[A^{*}\left(\alpha-\alpha^{-2}\right)\right]
$$

were plotted against reciprocal elongation, as suggested by the semiempirical equation of Mooney and Rivlin,

$$
\left[f^{*}\right]=2 C_{1}+2 C_{2} \alpha^{-1}
$$

in which $2 C_{1}$ and $2 C_{2}$ are constants independent of $\alpha^{19}$ Typical isotherms are shown in Figure 1. Several isotherms show an upturn in $\left[f^{*}\right]$ which is presumably due to strain-induced crystallization, as is generally ${ }^{15}$ (but not always) ${ }^{20,21}$ the case. The fact that the other isotherms showed no upturn indicates that at these values of the network chain length, rupture occurred before the reinforcing effects of the crystallites became large enough to be discernible. The decrease in $\left[f^{*}\right]$ just prior to its increase, which was very pronounced in one case, has been observed previously for other crystallizable polymer networks. ${ }^{14}$ Also, as expected, the desired elastic reversibility was obtained only for elongations below the value producing a discernible amount of crystallization. In any case, the only point of direct relevance here is the fact that there are sufficient data in all cases to provide values of the constants $2 C_{1}$ and $2 C_{2}$ characterizing the linear portions of the isotherms. These values were obtained by leastsquares analysis, and are given in columns five and six of the Table. The constant $2 C_{1}$ respresents the value of the modulus in the limit at large

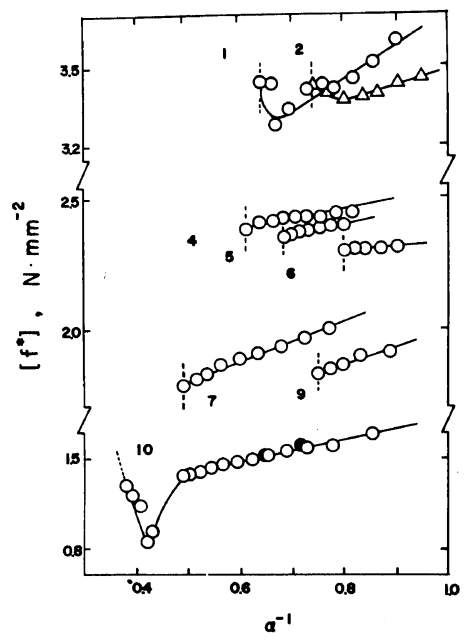

Figure 1. Representative stress-strain isotherms for the PCL networks in elongation at $25^{\circ} \mathrm{C}$; the labels on the curves are the isotherm designations in Table I. Results obtained using a series of increasing values of the elongation $\alpha$ are located by the open symbols. The other results, shown by the filled symbols, were obtained using values of $\alpha$ out of sequence in order to test for reversibility. All isotherms were located by leastsquare analysis, and the vertical lines locate the rupture points of the networks.

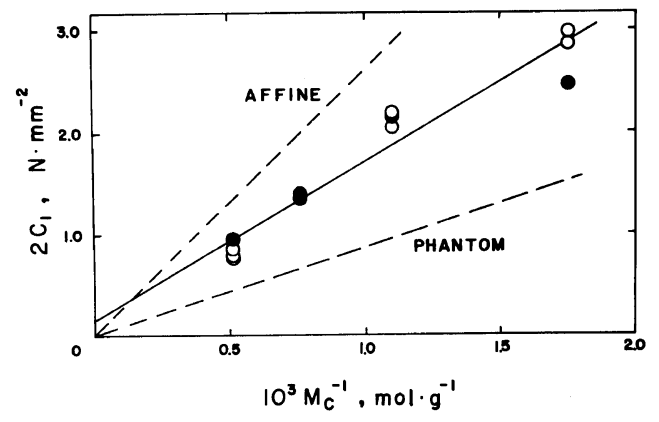

Figure 2. The modulus in the limit at very high elongation shown as a function of reciprocal molecular weight. The filled and unfilled circles refer to results obtained in the unswollen and swollen states, respectively. The two additional lines show the theoretical prediction for the non-affine deformation of a "phantom" network, and the affine deformation of a network in which the cross-links are firmly embedded in their surroundings. ${ }^{2,6}$

deformation $\left(\alpha^{-1} \rightarrow 0\right)$, and $2 C_{1}+2 C_{2}$ its value in the limit at small deformation $\left(\alpha^{-1} \rightarrow 1\right)$.

The values of $2 C_{1}$ are shown as a function of 
reciprocal molecular weight in Figure 2. The two theoretical curves shown were calculated from the equation $^{1-3}$

$$
2 C_{1}=A_{3} \rho R T v_{2 \mathrm{C}}^{2 / 3} M_{n}^{-1}
$$

in which $A_{3}$ is the "structure factor" for the trifunctional networks, $\rho$ is the density of the network, $R$ is the gas constant, and $T$ is the absolute temperature. The value of $A_{3}$ is predicted to be unity in the case of an affine deformation and $(1-2 / \phi)=1 / 3$ in the case of the very non-affine deformation associated with a phantom network. $^{2-6}$ The experimental results are seen to be intermediate to these two extremes. This suggests that at least over this molecular weight range, the network cross-links do undergo some fluctuations, but apparently not to the extent of those in a phantom network. It is also conceivable, of course, that some strain-induced crystallization occurred even in the linear portions of the isotherms, thereby increasing the observed values of $2 C_{1} \cdot{ }^{14} \mathrm{In}$ any case, the experimental values of $A_{3}$ calculated from eq 4 are given in column seven of the Table, and yield the results $A_{3}=0.66( \pm 0.06)$.

It is also of interest to consider the $2 C_{1}$ intercept, which corresponds to an infinite molecular weight between cross-links. Of particular importance is the comparison of the experimental value of the intercept with that predicted on the asumption that "inter-chain entanglements" which are "trapped" in a network structure contribute as effectively to the equilibrium modulus as they do to the plateau modulus observed in dynamic, viscoelastic measurements on the uncross-linked polymer. This would add to the modulus a contribution given by

$$
2 C_{1}=A_{4} \rho R T M_{\mathrm{en}}^{-1}
$$

where the entanglements are considered to be tetrafunctional and separated by the average molecular weight $M_{\mathrm{en}} \cdot{ }^{22,23}$ An experimental value of $M_{\mathrm{en}}$ for PCL is not available and, since this quantity does not correlate with structure in any obvious manner, it would be very difficult to predict one. Nonetheless, a reasonable guess would be that PCL has a value of $M_{\mathrm{en}}$ similar to that for poly-

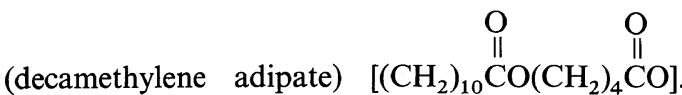
If $M_{\mathrm{en}}$ is approximated as one-half of the critical molecular weight for entangling, ${ }^{22}$ then its value would be $2500 \mathrm{~g} \mathrm{~mol}^{-1}$ Use of this value in eq 5 would predict a $2 C_{1}$ intercept of $0.16 \mathrm{~N}$ $\mathrm{mm}^{-2}$. The intercept observed from Figure 2 is only $0.16 \mathrm{~N} \mathrm{~mm}^{-2}$ and, as has already been mentioned, may be due to strain-induced crystallization. The relatively small value of this intercept supports the conclusion ${ }^{1,7,11,17}$ that interchain entanglements do not make very large contributions to the equilibrium modulus of a network.

In the case of the unswollen networks, it is also possible to provide a similar interpretation of the modulus in the limit at small deformation. The relevant structure factor $A_{3}^{\prime}$ is defined by ${ }^{24}$

$$
2 C_{1}+2 C_{2}=A_{3}^{\prime} \rho R T v_{2 \mathrm{C}}^{2 / 3} M_{n}^{-1}
$$

and should have values significantly larger than $A_{3}$; in the limit of a perfectly affine deformation, $A_{3}^{\prime}$ should be equal to unity. ${ }^{2-4}$ The experimental values for the four relevant isotherms gave $A_{3}^{\prime}=1.03$ $( \pm 0.22)$, in good agreement with theory. Thus, neither the high deformation nor the low deformation equilibrium moduli of the PCL networks provides any evidence for large contributions from inter-chain entanglements.

Acknowledgements. It is a pleasure to acknowledge the financial support provided by the National Science Foundation through Grant DMR 79-18903 (Polymers Program, Division of Materials Research), and the predoctoral fellowship awarded P.H. S. by the IBM Corporation, San Jose, CA. The authors are indebted to Drs. J. V. Koleske and D. H. Chadwick for samples and much useful information on the preparation of urethane-type networks.

\section{REFERENCES}

1. J. E. Mark, Makromol. Chem., 180 Suppl. 2, 87 (1979), and pertinent references cited therein.

2. P. J. Flory, Proc. R. Soc. London, Ser. A, 351, 351 (1976).

3. P. J. Flory, J. Chem. Phys., 66, 5720 (1977).

4. G. Ronca and G. Allegra, J. Chem. Phys., 63, 4990 (1975).

5. W. W. Graessley, Macromolecules, 8, 186 and 865 (1975).

6. P. J. Flory, Polymer, 20, 1317 (1979).

7. P.-H. Sung and J. E. Mark, manuscript submitted to J. Polym. Sci., Polym. Phys. Ed., and pertinent references cited therein. 
8. T. L. Smith and A. B. Magnusson, J. Appl. Polym. Sci., 14, 218 (1961).

9. D. J. Walsh, J. S. Higgins, and R. H. Hall, Polymer, 20, 951 (1979).

10. R. F. T. Stepto, Polymer, 20, 1324 (1979).

11. J. E. Mark and P.-H. Sung, to be published in Eur. Polym. J., 16, (1980).

12. C. G. Seefried, Jr., J. V. Koleske, F. E. Critchfield, and C. R. Pfaffenberger, J. Polym. Sci., Polym. Phys. $E d ., 18,817$ (1980).

13. J. V. Koleske and R. D. Lundberg, J. Polym. Sci., A2, 7, 795 (1969).

14. J. E. Mark, Polym. Eng. Sci., 19, 409 (1979).

15. J. E. Mark, Polym. Eng. Sci., 19, 254 (1979).

16. J. E. Mark and P. J. Flory, J. Appl. Phys., 37, 4635 (1966).
17. J. E. Mark and J. L. Sullivan, J. Chem. Phys., 66, 1006 (1977).

18. J. E. Mark, Rubber Chem. Technol., 48, 495 (1975).

19. M. Mooney, J. Appl. Phys., 19, 434 (1948); R. S. Rivlin, Phil. Trans. R. Soc. London, Ser. A., 241, 379 (1948).

20. A. L. Andrady, M. A. Llorente, and J. E. Mark, J. Chem. Phys., 72, 2282 (1980).

21. A. L. Andrady, M. A. Llorente, and J. E. Mark, J. Chem. Phys., 73, 1439 (1980).

22. J. D. Ferry, "Viscoelastic Properties of Polymers," 2nd ed, Wiley, New York, N.Y., 1970.

23. W. W. Graessley, Adv. Polym. Sci., 16, 1 (1974).

24. J. E. Mark and M. A. Llorente, J. Am. Chem. Soc., 102, 632 (1980). 